\title{
Gravitation induced shrinkage of Mercury's orbit
}

\author{
Qian Moxian, ${ }^{1}$ Li Xibin, ${ }^{1}$ and Cao Yongjun ${ }^{1, *}$ \\ ${ }^{1}$ College of physics and electronic information, Inner Mongolia Normal University, 010022 Hohhot, China
}

\begin{abstract}
In general relativity, the Mercury's orbit becomes approximately elliptical and the its perihelion has thus an additional advance. We demonstrate, meanwhile, that in comparison of those given by the Newton's theory of gravitation for the orbit of the Mercury, the circumference and the area are reduced by $40.39 \mathrm{~km}$ and $2.35 * 10^{9} \mathrm{~km}^{2}$, respectively, beside the major-axis contraction pointed out recently, and all are produced by the curved space within the Einstein's theory of gravitation. Since the resolution power of present astronomical distance measurement technology reaches one kilometer, the shrinkage of Mercury's orbit can then be observable.
\end{abstract}

\section{INTRODUCTION}

The gravitational field produced by the Sun causes the space nearby to be appreciably curved. Since the Mercury is the planet closest to the Sun, the general relativity provides a more accurate description of its orbit than what the Newton's law of universal gravitation does. The Mercury's perihelion advance, 43 arcseconds per century added to the planet's orbital precession, was very earliest successful prediction of the general relativity. [1-3] In fact, the advance has long been one of the observational cornerstones of general relativity, under more and more stringent theoretical examinations and experimental tests to date. [4,5] The gravitational interaction between the Mercury and the Sun does not only result in the perihelion advance of the orbit of Mercury, but also the radial contraction. [6] Recently, two new potentially observable effects on the orbit of Mercury are reported. [5,6] One is related to the angular coordinate, the second order effect of the perihelion advance, which adds 1 degree of arc every two billion years, believed to be detectable by BepiColombo, a European and Japanese mission to Mercury launched on 20 October 2018. [5] Another is related to the radial coordinate, the major-axis contraction of Mercury's elliptical orbit by an amount of $1.3 \mathrm{~km}$, which is within the limit of resolution power of current astronomical distance measurement technology which can be seen from the website of NASA where the distance of the Mercury from the Sun is updated every second with accuracy of one kilometer. [7, 8] We are confident that the major-axis contraction is one aftereffect of an overall shrinkage of the Mercury's orbit given by the general relativity in contrast to that given by the Newton's theory of gravitation. By an overall shrinkage of an ellipse, we mean that both the circumference and the area are reduced to some extents, though in general relativity the Mercury's orbit is approximately elliptical.

In Sec. II, the basics of the Mercury's orbit are outlined. In Sec. III, the circumference of the Mercury's orbit is explicitly calculated and illustrated to be shorter than that given by the Newton's theory. In Sec. IV, the area of the Mercury's orbit is defined and then shown to be less than that given by the Newton's theory. The last section V is a brief conclusion.

\section{BASICS OF THE MERCURY'S ORBIT}

In the Newton's law of universal gravitation, the space-time is flat, and the trajectory of a planet circling the Sun follows a perfect equation of the ellipse,

$$
R(\theta)=\frac{r_{\mathrm{gs}}}{b^{2}} \frac{1}{1+e \cos \theta},
$$

where $R$ is the radial coordinate in the plane polar coordinate system, $\theta$ is the angular coordinate, $e$ is centricity, $b=$ $G M / c L$ is a dimensionless characteristic quantity, $L$ is the angular momentum of a unit mass planet, $c$ is the speed of light, and $r_{\mathrm{gs}}=G M / c^{2} \approx 1.47 \mathrm{~km}$ is the Schwarzschild radius of the Sun. For the Mercury, $e=0.206$, and $b=1.63 * 10^{-4} \cdot[7,8]$

In the Einstein's gravitational theory, the space-time is curved, and the space in the solar system is described by

*Electronic address: phyjcao@imnu.edu.cn 
the Schwarzschild metric with the Sun at the origin $r=0$,

$$
d s^{2}=-\left(1-\frac{2 r_{\mathrm{gs}}}{r}\right) d t^{2}+\frac{d r^{2}}{1-2 r_{\mathrm{gs}} / r}+r^{2}\left(d \theta^{2}+\sin ^{2} \theta d \phi^{2}\right) .
$$

where $(r, \theta, \phi)$ are spherical polar coordinates, and $d s$ is the infinitesimal proper length element. It is evidently that once $r \gg r_{\mathrm{gs}}$, the space is asymptotically flat and only the orbit of the Mercury, the innermost planet in the solar system, is noticeably distorted from (1) as, [6]

$$
r \approx r_{\mathrm{gs}}\left[\frac{1}{b^{2}(1+e \cos t)}-\frac{2\left(3+2 e^{2}+e^{2} \cos t\right)}{(1+e \cos t)^{2}} \sin ^{2}\left(\frac{t}{2}\right)\right]=R(t)-r_{\mathrm{gs}} f(t),
$$

where

$$
f(t)=\frac{2\left(3+2 e^{2}+e^{2} \cos t\right)}{(1+e \cos t)^{2}} \sin ^{2}\left(\frac{t}{2}\right)
$$

and $t$ is a parameter related to angular variable $\theta$ via,

$$
t(\theta) \approx\left(1-3 b^{2}\right) \theta, \text { or, } \theta \approx\left(1+3 b^{2}\right) t(\theta) .
$$

The amount of the procession of Mercury's perihelion in a period $\Delta t=2 \pi$ is therefore,

$$
\Delta \theta \approx 3 b^{2} \Delta t(\theta)=6 \pi b^{2}
$$

It is the classic result [1-3] which in a century of the Earth gives 43 arcseconds. The second order angular procession is of order $O\left(b^{4}\right)=O\left(10^{-15}\right)$. [5] From relation (5), it is worth stressing that $t \in[0,2 \pi]$ is equivalent to $\theta \in\left[0,2 \pi+6 \pi b^{2}\right]$.

The infinitesimal line element $d \ell$ along the trajectory of the Mercury is in flat space

$$
d \ell=\sqrt{d x^{2}+d y^{2}}=\sqrt{d R^{2}+(R d \theta)^{2}}=\sqrt{R^{2}+\left(\frac{d R}{d \theta}\right)^{2}} d \theta .
$$

With $R$ given by (1) the circumference of Mercury's orbit in Newton's theory of gravitation is,

$$
\ell_{n}=\int_{0}^{2 \pi} \sqrt{R^{2}+\left(\frac{d R}{d \theta}\right)^{2}} d \theta
$$

Similarly, the area $S_{n}$ of the ellipse is,

$$
S_{n}=\frac{1}{2} \int_{0}^{2 \pi} R(\theta) d \ell=\frac{1}{2} \int_{0}^{2 \pi} R(\theta) \sqrt{\left(\frac{d R}{d \theta}\right)^{2}+R^{2}} d \theta .
$$

In Einstein's theory of gravitation, the circumference $\ell$ and the area $S$ must be defined by, respectively, the length of the trajectory of the Mercury and the area swept by a moving line connected the Mercury and the Sun, from one perihelion to the next one, from which the angular parameter $\theta$ changes from 0 to $2 \pi+6 \pi b^{2}$, i.e., $t$ changes from 0 to $2 \pi$ from (5), Two differences $\ell-\ell_{n}$ and $S-S_{n}$ define, respectively, the changes of the circumference and the area of Mercury's orbit.

\section{CALCULATION OF THE CIRCUMFERENCE DIFFERENCE $\ell-\ell_{n}$}

The space-time of around the Sun is static, and the spatially separated clocks are synchronized in metric (2). The infinitesimal proper distance element $d \ell$ along the circumference of the Mercury's orbit is from (2)

$$
d \ell^{2}=\frac{1}{1-2 r_{\mathrm{gs}} / r} d r^{2}+r^{2} d \theta^{2}
$$

i.e.,

$$
d \ell=\sqrt{\frac{1}{1-2 r_{\mathrm{gs}} / r}\left(\frac{d r}{d \theta}\right)^{2}+r^{2}} d \theta
$$


and

$$
\ell=\int_{0}^{2 \pi+6 \pi b^{2}} \sqrt{\frac{1}{1-2 r_{\mathrm{gs}} / r}\left(\frac{d r}{d \theta}\right)^{2}+r^{2}} d \theta=\int_{0}^{2 \pi} \sqrt{\frac{1}{1-2 r_{\mathrm{gs}} / r}\left(\frac{d r}{d t}\right)^{2}+r^{2}} d t
$$

To determine the length to the order of $\mathcal{O}\left(r_{\mathrm{gs}}\right)$, we need to expand $r^{2}$ and $(d r / d t)^{2}$ to this order. We have from $(3)$ and (5),

$$
\begin{aligned}
r^{2} & \approx R(t)^{2}-2 r_{\mathrm{gs}} R(t) f(t), \text { and } \\
\frac{d r}{d t} & \approx \frac{d R(t)}{d t}-r_{\mathrm{gs}} \frac{d f(t)}{d t}, \text { and } \\
\left(\frac{d r}{d t}\right)^{2} & \approx\left(\frac{d R(t)}{d t}\right)^{2}-2 r_{\mathrm{gs}} \frac{d R(t)}{d t} \frac{d f(t)}{d t}
\end{aligned}
$$

The approximate expression for $\left(1-2 r_{\mathrm{gs}} / r\right)^{-1}(d r / d t)^{2}$ to the order of $\mathcal{O}\left(r_{\mathrm{gs}}\right)$ is

$$
\frac{1}{1-2 r_{\mathrm{gs}} / r}\left(\frac{d r}{d t}\right)^{2} \approx\left(\frac{d R(t)}{d t}\right)^{2}-2 r_{\mathrm{gs}} \frac{d R(t)}{d t} \frac{d f(t)}{d t}+2 r_{\mathrm{gs}} \frac{1}{R(t)}\left(\frac{d R(t)}{d t}\right)^{2} .
$$

Combining two results (13a) and (14), we have

$$
\frac{1}{1-2 r_{\mathrm{gs}} / r}\left(\frac{d r}{d t}\right)^{2}+r^{2} \approx\left(\frac{d R(t)}{d t}\right)^{2}+R(t)^{2}-2 r_{\mathrm{gs}} Q(t),
$$

where

$$
Q(t)=\frac{d R(t)}{d t} \frac{d f(t)}{d t}+R(t) f(t)-\frac{1}{R(t)}\left(\frac{d R(t)}{d t}\right)^{2}
$$

We obtain

$$
\sqrt{\frac{1}{1-2 r_{\mathrm{gs}} / r}\left(\frac{d r}{d t}\right)^{2}+r^{2}} \approx \sqrt{\left(\frac{d R}{d t}\right)^{2}+R^{2}}-r_{\mathrm{gs}} \cdot \frac{Q(t)}{\sqrt{\left(\frac{d R}{d t}\right)^{2}+R^{2}}}
$$

Now we are in position to compute $\ell$ (12) which is given by

$$
\begin{aligned}
\ell & \approx \int_{0}^{2 \pi} \sqrt{\frac{1}{1-2 r_{\mathrm{gs}} / r}\left(\frac{d r}{d t}\right)^{2}+r^{2}} d t \\
& \approx \int_{0}^{2 \pi} \sqrt{\left(\frac{d R}{d t}\right)^{2}+R^{2} d t-r_{\mathrm{gs}} \int_{0}^{2 \pi} \frac{Q(t)}{\sqrt{\left(\frac{d R}{d t}\right)^{2}+R^{2}}} d t} \\
& \approx \ell_{n}-r_{\mathrm{gs}} \int_{0}^{2 \pi} \frac{Q(t)}{\sqrt{\left(\frac{d R}{d t}\right)^{2}+R^{2}}} d t .
\end{aligned}
$$

To get the difference $\Delta \ell \equiv \ell-\ell_{n}$ from above equation, we need to compute the last integration in it, which can be easily carried out numerically,

$$
\int_{0}^{2 \pi} \frac{Q(t)}{\sqrt{\left(\frac{d R}{d t}\right)^{2}+R^{2}}} d t \approx 27.48
$$

We find immediately

$$
\Delta \ell=-r_{\mathrm{gs}} \int_{0}^{2 \pi} \frac{Q(t)}{\sqrt{\left(\frac{d R}{d t}\right)^{2}+R^{2}}} d t \approx-27.48 r_{g s} \approx-40.39 \mathrm{~km} .
$$

Thus, the space curvature makes the circumference of Mercury's orbit shortened. 


\section{CALCULATION OF THE AREA DIFFERENCE $S-S_{n}$}

During one period $\theta \in\left[0,2 \pi+6 \pi b^{2}\right]$, a line drawn from Mercury to the Sun sweeps out an area as Mercury moves. The infinitesimal area element is defined by,

$$
d S=\frac{1}{2} r d \ell
$$

The formula of calculation of the area is then from (5),

$$
S=\frac{1}{2} \int_{0}^{2 \pi+6 \pi b^{2}} r d \ell(\theta)=\frac{1}{2} \int_{0}^{2 \pi} r d \ell(t) .
$$

In evaluation of this integral for the Mercury, we need to expand $r d \ell(t) / d t$ in it to order of $\mathcal{O}\left(r_{\mathrm{gs}}\right)$ from (12)

$$
\begin{aligned}
r \frac{d \ell(t)}{d t} & =r \sqrt{\frac{1}{1-2 r_{\mathrm{gs}} / r}\left(\frac{d r}{d t}\right)^{2}+r^{2}} \\
& \approx\left(R(t)-r_{\mathrm{gs}} f(t)\right)\left(\sqrt{\left(\frac{d R}{d t}\right)^{2}+R^{2}}-r_{\mathrm{gs}} \cdot \frac{Q(t)}{\sqrt{\left(\frac{d R}{d t}\right)^{2}+R^{2}}}\right) \\
& =R(t) \sqrt{\left(\frac{d R}{d t}\right)^{2}+R^{2}}-r_{\mathrm{gs}}\left(\frac{R(t) Q(t)}{\sqrt{\left(\frac{d R}{d t}\right)^{2}+R^{2}}}+f(t) \sqrt{\left(\frac{d R}{d t}\right)^{2}+R^{2}}\right) .
\end{aligned}
$$

The expression of the area $S$ to the order $\mathcal{O}\left(r_{\mathrm{gs}}\right)$ is then

$$
\begin{aligned}
S & \approx \frac{1}{2} \int_{0}^{2 \pi} R(t) \sqrt{\left(\frac{d R}{d t}\right)^{2}+R^{2}} d t-r_{\mathrm{gs}} \frac{1}{2} \int_{0}^{2 \pi}\left(\frac{R(t) Q(t)}{\sqrt{\left(\frac{d R}{d t}\right)^{2}+R^{2}}}+f(t) \sqrt{\left(\frac{d R}{d t}\right)^{2}+R^{2}}\right) d t \\
& \approx S_{n}-r_{\mathrm{gs}} G,
\end{aligned}
$$

where $r_{\mathrm{gs}} G$ is the first order correction from Einstein's theory with $G$ denoting an integral,

$$
G=\frac{1}{2} \int_{0}^{2 \pi}\left(\frac{R(t) Q(t)}{\sqrt{\left(\frac{d R}{d t}\right)^{2}+R^{2}}}+f(t) \sqrt{\left(\frac{d R}{d t}\right)^{2}+R^{2}}\right) d t
$$

which can be numerically determined to be

$$
G \approx 28.78 \frac{r_{\mathrm{gs}}}{b^{2}} .
$$

In final, we get the amount of the area reduction, $\Delta S=S-S_{n} \approx-r_{\mathrm{gs}} G$, with $b=1.63 * 10^{-4}$ and $r_{\mathrm{gs}}=1.47 \mathrm{~km}$, which is simply,

$$
\Delta S=-28.78 \frac{r_{\mathrm{gs}}^{2}}{b^{2}}=-2.35 * 10^{9} \mathrm{~km}^{2} .
$$

It is nearly the same area of a rectangle formed by one longer side length which is $57.91 * 10^{6} \mathrm{~km}$ which is the semimajor

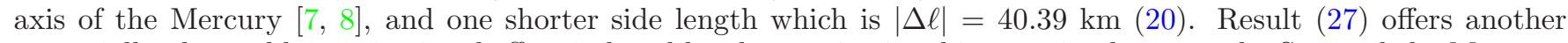
potentially observable gravitational effect induced by the gravitational interaction between the Sun and the Mercury.

\section{CONCLUSION}

It is an important issue to search for a new possible experimental hunt of a general relativistic effect. We report that the gravitational interaction between the Sun and the Mercury produces an overall shrinkage of the Mercury's orbit in comparison with that predicted by the Newton's theory of the gravitation. Explicitly, the circumference $C$ 
is reduced by an amount $40.39 \mathrm{~km}$, and the covering area $S$ of the orbit in a period is diminished by $2.35 * 10^{9} \mathrm{~km}^{2}$. With development of the modern technologies of measuring the sizes in astronomical scale, such a shrinkage seems to fall in the resolution power of the current apparatus.

[1] C. W. Misner, K. S. Thorne and J. A. Wheeler, Gravitation, (San Francisco: Freeman and Company. 1973).

[2] S. Weinberg, Gravitation and cosmology, (New York: Wiley. 1972).

3] L. D. Landau, E. M. Lifshitz, The Classical Theory of Fields. (4th ed.), (Oxford: Butterworth-Heinemann, 1998).

[4] A. M. Nobili, C. M. Will, Nature, 320(1986)39.

[5] C. M. Will, Phys. Rev. Lett. 120(2018)191101.

[6] Q. H. Liu, Q. Li, T. G. Liu and X. Wang, Mod. Phys. Lett. A 34(2019)1950159.

[7] NASA. Mercury, https://solarsystem.nasa.gov/planets/mercury/by-the-numbers/

[8] NASA, Mercury The Swiftest Planet, https:solarsystem.nasa.gov/planets/mercury/overview/. 